\title{
Ensino-Aprendizagem com Abordagem Colaborativa: aliando técnica pedagógica com Tecnologias Digitais de Informação e Comunicação
}

\author{
Winston Igor Severiano de Almeida ${ }^{1}$, Aladir Ferreira da Silva Júnior ${ }^{2}$ \\ ${ }^{1,2}$ Instituto de Federal de Educação, Ciência e Tecnologia de Goiás (IFG) - Câmpus Jataí \\ Núcleo de Informática na Educação (NINE) - 75.804-714 - Jataí - GO - Brasil \\ 1winstonigor14@gmail.com, 2aladir.juniordifg.edu.br
}

\begin{abstract}
For some time now, the actors involved in teaching and learning have been using Digital Information and Communication Technologies (TDIC) to enhance their actions. However, it is well known that it is not enough to use TDIC, it is necessary to combine it with an appropriate pedagogical technique. This article presents the report on the use of the collaborative technique called the Verbalization Group/Observation Group $(G V-G O)$, together with the Google Suite. The technique was applied in a higher technology course with students of Algorithms II. It was possible to conclude that the transposition of the GV-GO technique for use with TDIC brought benefits specially regarding the spatial organization of the students. The analysis of data collected from the students indicated acceptance and relevance in the use of the technique in the classroom/laboratory.
\end{abstract}

Resumo. Já há algum tempo os atores envolvidos no ensino-aprendizagem têm usado as Tecnologias Digitais de Informação e Comunicação (TDIC) para potencializar suas ações. No entanto, é notório que não basta usar as TDIC, é preciso aliá-las à uma técnica pedagógica apropriada. Apresenta-se neste artigo o relato de uso da técnica colaborativa chamada Grupo de Verbalização/Grupo de Observação (GV-GO), aliada à Suite Google. A técnica foi aplicada em um curso superior de tecnologia com alunos da disciplina de Algoritmos II. Foi possível concluir que a transposição da técnica GVGO para uso com TDIC trouxe beneficios especialmente quanto à organização espacial dos discentes. A análise de dados coletados junto ao alunado indicou aceitação $e$ relevância no uso da técnica em sala de aula/laboratório.

\section{Introdução}

Com o avanço, a olhos vistos, das Tecnologias Digitais de Informação e Comunicação (TDIC), as informações estão cada vez mais acessíveis no mundo digital. No âmbito do ensino-aprendizagem as TDIC trazem consigo novas possibilidades de se (re)pensar o aprender e o ensinar. Uma TDIC pode ser compreendida como um recurso tecnológico moderno como um computador, tablet, smartphone, e qualquer outro dispositivo que permita a navegação na internet [Baranauskas e Valente, 2013].

Em contraposição a um modelo de aprendizagem centrado no professor, algumas abordagens têm sido bastante discutidas [Torres e Irala, 2014] e primordialmente propugnam a colocar o aprendiz como protagonista do seu próprio processo de ensino-aprendizagem. Uma dessas abordagens que fomentam atividades de colaboração na aprendizagem em grupo é a Aprendizagem Colaborativa (AC). 
Embora as TDIC estejam disponíveis em maior escala e diversas abordagens de ensino-aprendizagem estejam discutidas na literatura há algum tempo, percebe-se que nem sempre isso é aplicado no ambiente formal de aprendizagem. Diversos fatores podem contribuir para essa não-adoção: (i) a indisponibilidade de equipamentos, softwares e conectividade no ambiente escolar, (ii) a falta de formação/capacitação do professor para criação/uso das TDIC, (iii) a falta de apoio da gestão escolar, (iv) a indiferença por parte dos alunos, dentre outros.

Cada um dos fatores apontados são importantes para uma efetiva adoção das TDIC no ensino-aprendizagem. No lócus desta pesquisa, os fatores (i) e (iii) têm menor peso, haja vista que a instituição dispõe de equipamentos e softwares em diversos laboratórios, com conectividade tanto local como acesso a Internet e a gestão tem envidado esforços no sentido de possibilitar capacitação aos professores e alunos no uso dessas tecnologias. Em relação ao item (ii) apesar de cursos oferecidos pela instituição, a formação é tema complexo e deve ser continuada dada a características dinâmicas das TDIC.

Em levantamento inicial, realizado a partir de conversas informais com professores da instituição e também pela vivência dos pesquisadores, a maior dificuldade apontada foi que tanto as formações oferecidas pela gestão como àquelas que procuraram por iniciativa própria na Internet, são voltadas para capacitação em uma ou outra ferramenta tecnológica, porém não se aborda como aliar seu uso com uma técnica pedagógica.

Percebemos que esse fator pode trazer alguma insegurança ao professor, que no pouco tempo disponível ao exercício de seu mister precisa buscar uma técnica que possa ser adequada à sua turma/conteúdo e ainda aliá-la a uma TDIC. A partir dessa lacuna nesta pesquisa se objetivou organizar algumas técnicas pedagógicas, com foco na aprendizagem colaborativa, que aliadas a alguma(s) TDIC pudessem auxiliar o professor em seu trabalho. A hipótese é que ao aliar TDIC e técnicas pedagógicas colaborativas a motivação dos alunos seja despertada, o que contribuiria para resolver o item (iv) - a indiferença por parte dos alunos.

\section{Técnicas Pedagógicas Colaborativas}

Diversas técnicas pedagógicas colaborativas podem ser encontradas na literatura. No âmbito desta pesquisa foram transcritas oito delas, a saber: (i) Phillips-66, (ii) Grupo de Verbalização/Grupo de Observação (GV-GO), (iii) Grupo de Cochicho, (iv) Solução de Problemas Estruturados, (v) Envio de um Problema, (vi) Estudo de Caso, (vii) Edição em Pares, (viii) Escrita Colaborativa. Para fins de clareza, neste artigo apresentar-se-á com mais detalhes apenas a técnica colaborativa GV-GO.

\subsection{Técnica Colaborativa GV-GO}

A estratégia GV-GO é uma das técnicas de ensino-aprendizagem flexível podendo ter o seu uso adaptado indo de acordo com número de alunos na sala de aula. A dinâmica da técnica ocorre na realização de atividades a partir de grupos, seguindo um tempo estipulado pelo o docente [Anastasiou e Alves, 2004].

A técnica é baseada na divisão de dois grupos de alunos, caracterizando em um grupo de verbalização $(\mathrm{GV})$, responsável por estudar/debater/apresentar o conteúdo proposto pelo professor, e um grupo de observação (GO) responsável por observar e anotando toda a discussão realizada pelo o grupo GV. Logicamente que o professor poderá criar quantas duplas de grupos quiser. Após esgotar o tempo estipulado pelo orientador da atividade, os 
grupos invertem o papel, ou seja, o grupo GV passa a ser o GO, e o grupo que era GO passa a ser GV [Saldanha et al., 2019].

Para Anastasiou e Alves (2004), a estratégia GV-GO é útil quando o objetivo é fazer com que os grupos de discentes desenvolvam o conhecimento sobre um determinado assunto, elevem os níveis de habilidades intelectuais e verbais, e ainda, realizem a permuta de ideias em conjunto com os demais alunos. A técnica pode auxiliar os alunos a utilizarem operações do pensamento, às quais podem ser "[...] análise, interpretação, crítica, obtenção e organização de dados, comparação, resumo, e observação" [Saldanha et al. 2019, p. 8].

A estratégia GV-GO pode ser benéfica quando a necessidade do docente é fazer com que os alunos tenham interesse em analisar um determinado assunto ou problemática com alguma profundidade, discutir um problema buscando uma solução. Ainda é recomendada quando há interesse do professor em estimular o trabalho colaborativo e o desenvolvimento da auto-liderança [Saldanha et al., 2019].

\section{Métodos}

Para a consecução desta pesquisa aplicada, se procedeu inicialmente a um levantamento dos Projetos Pedagógicos de Curso (PPC) dos cursos de Bacharelado em Engenharia Civil, Bacharelado em Engenharia Elétrica e Tecnologia em Análise e Desenvolvimento de Sistemas (TADS), com a finalidade de verificar se nestes documentos havia referência à previsão de uso de TDIC. Adicionalmente, se realizou uma pesquisa junto ao alunado destes cursos, dentre eles trinta e cinco responderam por meio de um questionário digital com a finalidade de corroborar ou refutar as informações fornecidas em conversas informais acerca do uso das TDIC em sala de aula ou laboratórios. Também se realizou uma pesquisa de prospecção com professores que atuam nos três cursos elencados.

Foi feita análise dos resultados apresentados nas pesquisas de levantamento dos PPC, bem como as com alunos e professores e a partir disso verificou-se que não havia uma presença maciça das TDIC na prática de sala de aula/laboratório, mas sim algumas iniciativas do professor em trabalhar com estas tecnologias de forma experimental.

A partir do aprofundamento do conhecimento dos pesquisadores sobre a Aprendizagem Colaborativa realizado nas pesquisa de [Oliveira et al., 2019], [Torres e Irala, 2014], foi realizado um estudo das técnicas pedagógicas que trabalham com essa abordagem nos documentos de [Barkley et al., 2014], [Anastasiou e Alves, 2004] e outros, objetivando elencar um conjunto de técnicas para compor um Guia de apoio ao professor em suas respectivas disciplinas. A descrição de passos de oito técnicas pedagógicas colaborativas foram adaptadas para uso com suporte as TDIC disponibilizadas pela suíte de aplicativos da Google. Esse trabalho foi realizado com o apoio de um professor com experiência em TDIC, além do pesquisador.

O motivo de escolher a suíte Google foi principalmente por ser gratuita, permitir atividades colaborativas síncronas, ter integração entre as suas ferramentas (p. ex. editor de texto, planilha eletrônica, formulários, editor de apresentações entre outros recursos). Adicionalmente, estas ferramentas com recursos avançados estão disponíveis por meio de convênio entre a Google e a instituição onde essa pesquisa foi realizada.

Por fim foram realizadas sessões com atividades práticas, com a finalidade de trazer subsídios sobre o uso/aceitação das técnicas pedagógicas transpostas para uso com TDIC. 


\section{Resultados e Discussão}

Esta seção foi organizada em três subseções, a saber: (4.1) Transposição da GV-GO para uso conjunto com uma TDIC, (4.2) Uso prático em sala de aula/laboratório e (4.3) Pesquisa de aceitação de uso da técnica junto aos alunos. Em cada seção serão apresentados e discutidos os resultados obtidos.

\subsection{Transposição da técnica pedagógica GV-GO com o uso de uma TDIC}

Para o uso da técnica com a TDIC foi realizado uma análise a partir da descrição dos passos a serem seguidos por professores e alunos, de acordo com o que propugna a técnica e a partir disso foram realizadas as adaptações necessárias. É importante ressaltar que as adaptações realizadas com o intuito de permitir ao professor fazer o uso da GV-GO junto a TDIC, não altera a essência desta técnica.

De modo similar aos passos que compõem um algoritmo de computador, foi possível encontrar na literatura os passos que constituem o algoritmo da GV-GO, descritos a seguir, por meio da sigla $P$ seguida de um numeral para apresentar a sequência dos passos.

P-1: O professor deve propor que o alunos se organizem em grupos, levando em consideração que deve haver no mínimo dois grupos;

P-2: O professor deve expor o(s) problema(s) a ser(em) resolvido(s) e explica aos alunos quais os objetivos que busca alcançar com o grupo de alunos com essa atividade;

P-3: O professor deve explicar aos alunos como o processo de discussão ocorrerá;

P-4: Com os grupos já formados, o professor deverá instruir os alunos de modo que o grupo caracterizado como Grupo de Verbalização (GV) forme um círculo, enquanto o outro grupo, nomeado de Grupo de Observação (GO), forme outro círculo, este ao redor do grupo $\mathrm{GV}$;

P-5: O professor deve estabelecer, informar e controlar o tempo para que o grupo GV faça as suas ponderações sobre a problemática. Para o grupo GO, o professor deve orientá-los para que observem e façam comentários referentes às informações apresentadas pelo o grupo GV e os anote;

P-6: O grupo GV deve começar a realizar o debate/apresentação ao mesmo tempo que o grupo GO observa atentamente e realiza as devidas anotações;

P-7: Considerando o tempo esgotado, o professor deve orientar a necessidade da troca de funções, ou seja, o grupo que atua como GO passa a atuar como GV, e um novo debate/apresentação deve acontecer, ou seja, retorne ao Passo 6;

P-8: Após todos os alunos terem participado das discussões, o professor deve realizar uma conclusão/avaliação do que foi discutido em sala de aula.

Para utilização junto ao GV-GO, foram analisadas e escolhidas as ferramentas Google Documentos, editor de textos síncrono, e o Google Apresentações, editor síncrono de apresentações eletrônicas. As duas ferramentas foram escolhidas pois além de serem de uso genérico, possuem os mesmos recursos como a possibilidade do trabalho simultâneo, e permitem ao professor se comunicar com os alunos por meio delas.

Assim, a partir de cada passo do algoritmo da técnica se apresenta o uso da TDIC específica com a sua função para alcançar o objetivo do passo. Como resultado se obteve a 
seguinte transposição, que será apresentada no quadro 1 com a sigla TP, seguida de um numeral para explicitar a ordem. Ressalta-se que isso se fez necessário pois a transposição dos passos da técnica não resulta necessariamente em um mapeamento direto (1:1), mas pode resultar em um número maior (n:1) ou menor (1:n) de passos.

\section{Quadro 1 - Descrição de uso da técnica pedagógica GV-GO com TDIC} \begin{tabular}{l|l} 
TP-1 & $\begin{array}{l}\text { O professor cria um documento com o enunciado da tarefa/atividade no editor de textos síncrono } \\
\text { para cada um dos grupos e o compartilha com os seus membros. }\end{array}$
\end{tabular}

Percebe-se que nesse passo, apesar de o professor poder permitir que os alunos escolham com quem irão trabalhar, é responsabilidade do docente dar a orientação inicial, criando o arquivo e compartilhando o mesmo com os membros de cada um dos grupos. $\mathrm{O}$ aluno ao receber o link via e-mail tem acesso para edição no arquivo e já poderá trabalhar colaborativamente com seus colegas.

TP-2 O professor utilizando-se do bate-papo (chat) disponível no próprio editor, em caso de uso remoto, ou mesmo de forma convencional, expõe o problema a ser resolvido bem como os objetivos, em caso de uso presencial.

Com poucas mudanças em relação à P-2, apesar de que pela própria característica da ferramenta Google Docs é possível ampliar o espaço da sala de aula/laboratório permitindo que o aluno e o professor possam estar em espaços físicos distintos. O mesmo ocorre com P-3, onde o professor explicitará como a discussão entre os grupos, agora com o uso da TDIC, poderá ocorrer. No passo P4 se aborda a questão de como os grupos devem espacialmente ficarem organizados, $(\mathrm{GV})$ em um círculo interno e $(\mathrm{GO}) \mathrm{em}$ outro círculo ao redor do grupo GV. Verifica-se que com a transposição da técnica GV-GO essa organização já foi realizada no passo TP-2 também, pois o professor ao atribuir o documento com o enunciado a cada um dos membros já realizou os limites necessários para cada um dos grupos. A preocupação com a organização em círculos só faz sentido no contexto presencial sem o uso das TDIC relatadas.

TP-3 A resolução da tarefa deverá ser feita entre os membros de cada grupo, no mesmo arquivo compartilhado pelo professor. O professor deverá informar que, as dúvidas podem ser sanadas por meio do chat presente na TDIC.

O uso de qualquer ferramenta atrapalha a integração dos dados coletados, analisados e descritos, usando a TDIC apresentada aqui, os membros do grupo trabalham no mesmo arquivo, onde todos estão vendo o que está acontecendo de forma síncrona. Cabe ressaltar também que a TDIC fornece um histórico de todas as contribuições realizadas no arquivo compartilhado, o que pode ser uma fonte relevante de dados para avaliação pelo professor.

TP-4 O professor deve informar aos grupos, que eles têm à disposição a ferramenta de apresentações existente no pacote Google Docs para auxiliar no suporte ao debate, que deverá compreender os principais conceitos utilizados na solução do problema.

A diferença entre esse passo e o convencional (sem o uso da TDIC), basicamente se caracteriza pelo canal de comunicação, especialmente em caso de encontros não presenciais. Em relação à TDIC de apresentação o destaque é a possibilidade de se realizar uma atividade colaborativa, já que ela mantém as mesmas características do editor síncrono, histórico, bate-papo, entre outros.

TP-5 O professor deve cronometrar o tempo e solicitar que um dos grupos comece com a apresentação. Todos os demais serão grupos de observação (GO) nesse momento, observando atentamente e realizando as devidas anotações, usando a TDIC de editor síncrono. Para isso o professor compartilhou o arquivo gerado pelo grupo de verbalização (GV). 


\subsection{Uso prático em sala de aula/laboratório}

Com o intuito de verificar na prática como seria o uso da técnica GV-GO com o uso de TDIC, foi organizado um experimento com duas turmas dos cursos de Bacharelado em Engenharia Elétrica e Tecnologia em Análise e Desenvolvimento de Sistemas (TADS). Os resultados apresentados são semelhantes e por ordem de clareza neste artigo apresentar-se-á o experimento proposto à turma do segundo período do curso de TADS, em dois encontros no espaço cedido pelo professor da disciplina de Algoritmos-II.

O experimento ocorreu em laboratório de informática, disponibilizado pela instituição contato com 30 computadores ligados à Internet onde ocorrem costumeiramente as aulas práticas das disciplinas relacionadas à programação de computadores, presente em todas as matrizes curriculares dos cursos superiores da instituição.

Para que fosse possível a utilização da técnica GV-GO com experimentação prática, esclareceu-se ao professor para que, previamente conhecesse a técnica, os passos que a técnica propõe para o uso, analisasse e conhecesse quais eram os seus principais objetivos de modo a alinhá-los aos objetivos de sua aula. Ressalta-se que as noções da técnica foram apresentadas por meio de um e-book produzido pelo pesquisador e com a supervisão deste. Esse conhecimento basal da técnica permitiu ao professor escolher um conteúdo que julgouse adequado ser trabalhado com o uso da GV-GO.

O docente planejou a aplicação do GV-GO com a TDIC em sua disciplina/conteúdo visando atingir os seguintes objetivos: (i) permitir que os alunos compreendam os conceitos de modularização; (ii) possibilitar aos discentes o conhecimento de como passar parâmetros por valor e por referência na linguagem de programação $C$; (iii) entender o que é e como funciona o escopo de variáveis - locais e globais e (iv) assimilar o conceito de recursividade na programação em linguagem $\mathrm{C}$. 
Ao iniciar a aula, foi explicado aos alunos naquela ocasião, que eles iriam trabalhar colaborativamente em grupos com a capacidade de até três integrantes. A formação dos grupos ocorreu pelo compartilhamento de arquivos da ferramenta Google Documentos, conforme proposto em um e-book que apresenta a transposição de técnicas pedagógicas colaborativas para uso com TDIC, voltado especificamente para o professor.

Cada documento criado e compartilhado pelo professor continha a devida problemática para que os grupos buscassem a solução. $\mathrm{O}$ docente solicitou à turma que mediante sua requisição, fornecessem o endereço de suas respectivas contas Google, e assim foram inseridos até três alunos por documento, formando seis grupos.

À medida que os alunos iam repassando o seu endereço de correio eletrônico, de imediato iam recebendo o seu convite de acesso ao documento e, consequentemente, já podiam ter acesso a problemática e iniciar a comunicação entre os membros de seu grupo à busca de solução. $\mathrm{O}$ docente informou à turma que teriam o tempo de trinta minutos para solucionarem o problema.

No decorrer da atividade, à medida que algumas dúvidas iam surgindo, o docente prestava assistência via ferramenta de bate-papo (chat) disponível na própria TDIC, o Google Documentos. Essa funcionalidade se mostrou útil não apenas para dúvidas junto ao professor, mas também para que os próprios membros do grupo pudessem se comunicar, haja vista que eles não estavam juntos fisicamente. Percebeu-se o chat integrado à TDIC como um diferencial da ferramenta pois permite evidenciar a colaboração.

Após o término do tempo estipulado para a realização do primeiro momento da atividade, o professor reforçou as orientações à turma de como iria ocorrer o segundo momento, as apresentações das soluções encontradas pelos grupos. Se durante o primeiro momento somente os alunos do mesmo grupo debateram e geraram soluções descrevendo-as no Google Documentos, no segundo momento após a apresentação se possibilitou um debate coletivo acerca das soluções apresentadas por cada grupo de verbalização, já que todos os outros grupos eram observadores no momento.

A técnica GV-GO propõe que os alunos façam um debate, referente aos resultados alcançados, com toda a turma. Com o intuito de auxiliar na coordenação dessa atividade, o professor destacou a possibilidade de uso de outra ferramenta escalada para ser utilizada junto a técnica de ensino-aprendizagem. O Google Apresentações foi utilizado pelos grupos para prepararem suas apresentações, e expondo soluções encontradas.

Durante o processo de análise e busca de soluções no primeiro momento da técnica visto na figura 1(a) é relevante observar que, apesar de cada aluno estar em seus respectivos grupos, eles não estão juntos, em círculos como originalmente propõe a técnica. Percebe-se que a TDIC escolhida atua como um catalisador, favorecendo a flexibilidade espacial na aplicação da GV-GO. Agora, com o acontecimento do segundo momento da técnica com o uso de TDIC, ocorreu a mudança de características dos grupos e iniciaram-se as apresentações. No decorrer das apresentações o grupo que estava a frente ainda era considerado como grupo GV visto na figura 1(b). O grupo GV apresenta as suas soluções com a utilização da ferramenta Google Apresentações enquanto o grupo GO visto na figura 1(c) observa elencando possíveis ponderações.

Após todos os grupos terem exposto e debatido as suas soluções, o professor fez as considerações finais. Este momento foi importante para que os alunos pudessem indagar ao professor dúvidas não esclarecidas durante a atividade. 


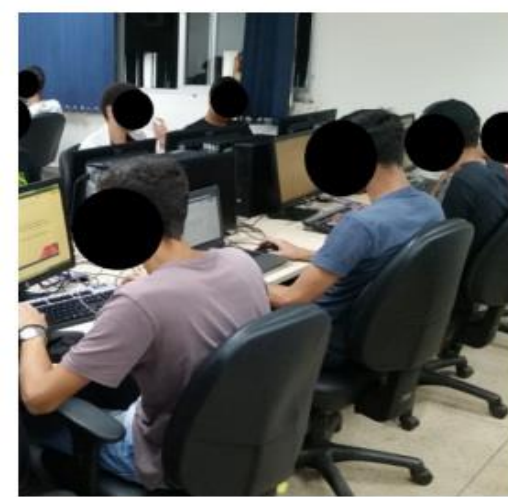

Figura 1(a) - formação GV/GO



Figura 1(b) GV apresentando

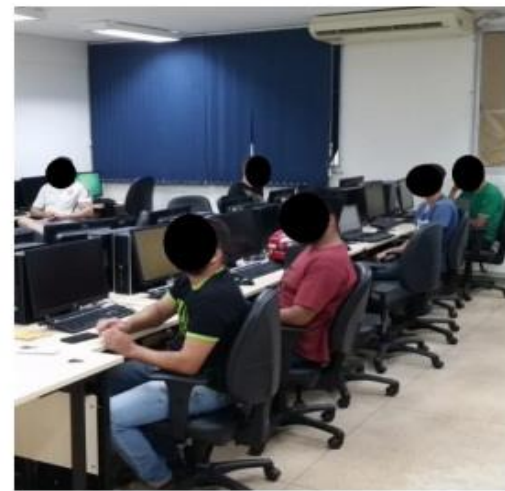

Figura 1(c) GO observando

\subsection{Pesquisa de aceitação de uso da técnica junto aos alunos}

Com a finalidade de se analisar as considerações quanto ao uso da técnica pedagógica adaptada e aplicada como estratégia de ensino na disciplina de Algoritmos-II, foi solicitado aos alunos que respondessem a um questionário online. $\mathrm{O}$ questionário foi respondido por doze alunos após a realização da atividade e fundamentou-se em relação aos conteúdos e também às percepções que os alunos tiveram sobre o uso da técnica.

Apesar da disciplina de Algoritmos ser fundamental para o ensino da programação de computadores, seus conteúdos são vistos como barreiras para os estudantes [Machado, 2018]. Buscando verificar a existência dessas dificuldades, uma das questões solicitava que o aluno marcasse em quais conteúdos ele sentia maior dificuldade. Foram, portanto, apresentados os conteúdos a partir da ementa da disciplina.

Como resultados obteve-se que o conceito de modularização (funções e procedimentos), apresentou dificuldade para 46,2\% dos alunos. $61,5 \%$ dos participantes informou que o conteúdo de recursividade também apresenta desafio à aprendizagem. A partir desses resultados e considerando que o conteúdo de modularização foi trabalhado durante o experimento com os alunos, um questionamento foi feito para verificar se os alunos perceberam algum ganho com o uso da técnica sobre o conteúdo estudado. 53,8\% dos alunos informaram que frequentemente puderam observar o ganho de conhecimento, 30,8\% que sempre obtiveram ganho de conhecimento, 7,7\% informou que às vezes e 7,7\% raramente.

Ao serem questionados se "a atividade realizada em grupo colaborou para melhor entendimento e aprendizado no conceito em questão? Por que? ", foram unânimes em responder que a técnica junto com a TDIC, contribuíram para o seu aprendizado, tendo como algumas respostas às seguintes: "Sim. Porque a interação com os colegas e a troca de experiência faz com que melhorou nosso aprendizado". "Sim, pois houve uma comunicação maior entre alunos, compartilhando ideia e modos de fazer", "Sim, pelo o fato dos alunos ter a oportunidade de testar se realmente dominava o conteúdo" e "Sim, pois os próprios alunos fazem uma pesquisa sobre o conteúdo, e ensinando é a melhor forma de aprender".

É importante ressaltar que, ainda como resultados do questionário $61,5 \%$ dos alunos não conheciam a técnica GV-GO, 23,1\% conheciam e 15,4\% disseram não ter certeza. No entanto, a maioria considera ser viável aplicar a estratégia para o ensino-aprendizagem dos conteúdos da disciplina de Algoritmos II, conforme pode ser visto no gráfico 1. 




Sempre

Frequentemente

As vezes

Raramente

Nunca

Gráfico 1 - Viabilidade esperada da GV-GO em Algoritmos II

\section{Conclusão}

Esta pesquisa é integrante de um projeto maior que visa propiciar ao docente uma coletânea de estratégias pedagógicas colaborativas com indicações de uso aliadas à TDIC, haja vista as possíveis dificuldades enfrentadas por esse profissional quando do exercício de seu mister, especialmente quanto ao tempo para levantar as técnicas e depois verificar a possibilidade de uso conjunto com as TDIC disponíveis.

Por esta pesquisa foi possível concluir que a transposição da técnica GV-GO para uso com TDIC trouxe benefícios. Se por um lado existia a necessidade de se ter uma organização em círculos e, portanto, dependeria de uma (re)organização do espaço onde a aula ocorreria, com a aplicação da TDIC isso pôde ser desprezado, já que a ferramenta cumpre o papel de aglutinar os membros do grupo virtual.

As técnicas e TDIC por si só não são panacéia para a solução dos problemas de ensino-aprendizagem. Daí vemos que os resultados dessa pesquisa podem, em certa medida, colaborar para que o professor tenha acesso à prescrições que podem encorajá-lo a utilizar de modo mais efetivo as TDIC. Esses resultados ficam ainda mais evidentes com o momento que a humanidade está vivendo, onde se recorre emergencialmente ao ensino remoto para o atendimento das necessidades educacionais, em virtude da pandemia da COVID-19.

Apesar dos resultados promissores tanto na estruturação da técnica usando TDIC, quanto pela aplicação direta em sala de aula/laboratório de informática, sabe-se que ainda há fatores limitantes para o pleno uso do proposto aqui. O conhecimento prévio sobre a TDIC, a disponibilidade de computadores e o acesso a Internet são alguns destes.

Em prosseguimento à esta pesquisa pretende-se aumentar o repertório de técnicas dispostas no e-book, verificar o uso dessas técnicas com o uso prático em sala de aula/laboratório e prospectar também outras TDIC além das avaliadas.

\section{Agradecimentos}

Agradecimentos ao Instituto Federal de Goiás por possibilitar o desenvolvimento da pesquisa e ao grupo de pesquisas NINE (http://www.nine.inf.br), que desenvolve um projeto âncora do qual esta pesquisa faz parte.

\section{Referências}

Anastasiou, L. D. G. C., \& Alves, L. P. (2004). Estratégias de ensinagem. Processos de ensinagem na universidade. Pressupostos para as estratégias de trabalho em aula, 3, 67-100. 
IX Congresso Brasileiro de Informática na Educação (CBIE 2020)

Anais do XXVI Workshop de Informática na Escola (WIE 2020)

Baranauskas, M. C. C., \& Valente, J. A. (2013). Editorial. Tecnologias, Sociedade e Conhecimento1(1),1-5.

https://www.nied.unicamp.br/revista/index.php/tsc/article/view/118/96

Barkley, E. F., Cross, K. P., \& Major, C. H. (2014). Collaborative learning techniques: A handbook for college faculty. John Wiley \& Sons.

Machado, L. D. P., Berkenbrock, C. D. M., Anselmo, G., \& Siple, I. Z. (2018). Uma ferramenta colaborativa para apoiar a aprendizagem de programação de computadores. Revista Brasileira de Computação Aplicada, 10(1), 23-29.

Oliveira, L., Rosa, S., \& Pimentel, A. (2019). Revisão Sistemática da Literatura: Formação de Grupos na Aprendizagem Colaborativa com Suporte Computacional. Brazilian Symposium on Computers in Education (Simpósio Brasileiro de Informática na Educação SBIE), 30(1), 1955.

Saldanha, G. C. B., Guerra, M. H. F. S., da Silva Firmino, E., Vasconcelos, A. K. P., \& de Goes Sampaio, C. (2019). Use of text and GV/GO study as Radioactivity teaching strategies. Research, Society and Development, 8(7), 21871123.

Torres, P. L., \& IRALA, E. A. F. (2014). Aprendizagem colaborativa: teoria e prática. Complexidade: redes e conexões na produção do conhecimento. Curitiba: Senar, 61-93. 